Special issue of the International Conference on Computational and Experimental Science and Engineering (ICCESEN 2014)

\title{
Monte Carlo Simulation of Photoneutron Dose in Radiotherapy Room as A Function of Gantry Angles
}

\author{
Ü. KARA ${ }^{a *}$, A. MesBahi ${ }^{b}$, I. AKKURT ${ }^{c}$ \\ ${ }^{a}$ Suleyman Demirel University, Vocational School of Healt Services, Isparta, Turkey \\ ${ }^{b}$ Medical Physics Department, Medical School, Tabriz University of Medical Sciences, Iran \\ ${ }^{c}$ Suleyman Demirel University, Physics Department, Isparta, Turkey
}

\begin{abstract}
Using bremsstrahlung photon is one of the principal means to treat cancer. Bremsstrahlung photons are created in medical LINAC and the interaction of bremsstrahlung photon with the heavy nuclei that constitute the linac head shielding is the main process of neutron production inside the treatment rooms. The threshold of the neutron production for those materals is about $10 \mathrm{MeV}$. As the neutron is more dangerous than photon it is important to determine neutron dose during radiotherapy treatment. The neutron distribution depends on some parameters such as gantry angle. In this study neutron dose rate has been simulated as a function of gantry angle.
\end{abstract}

DOI: 10.12693/APhysPolA.128.B-378

PACS: 87.10.Rt, 87.16.af

\section{Introduction}

Using bremsstrahlung photon is one of the main way to treat cancer cell. Bremsstrahlung photons are created in medical LINAC and the interaction of bremsstrahlung photon with the heavy nuclei that constitute the linac head shielding is the main process of neutron production inside the treatment rooms. The threshold of the neutron production for those materals is about $10 \mathrm{MeV}$. As the neutron is more dangerous than photon it is important to determine neutron dose during radiotherapy treatment. The neutron distribution depends on some parameters such as gantry angle. In this study neutron dose rate has been measured and simulated in radiotherapy room.
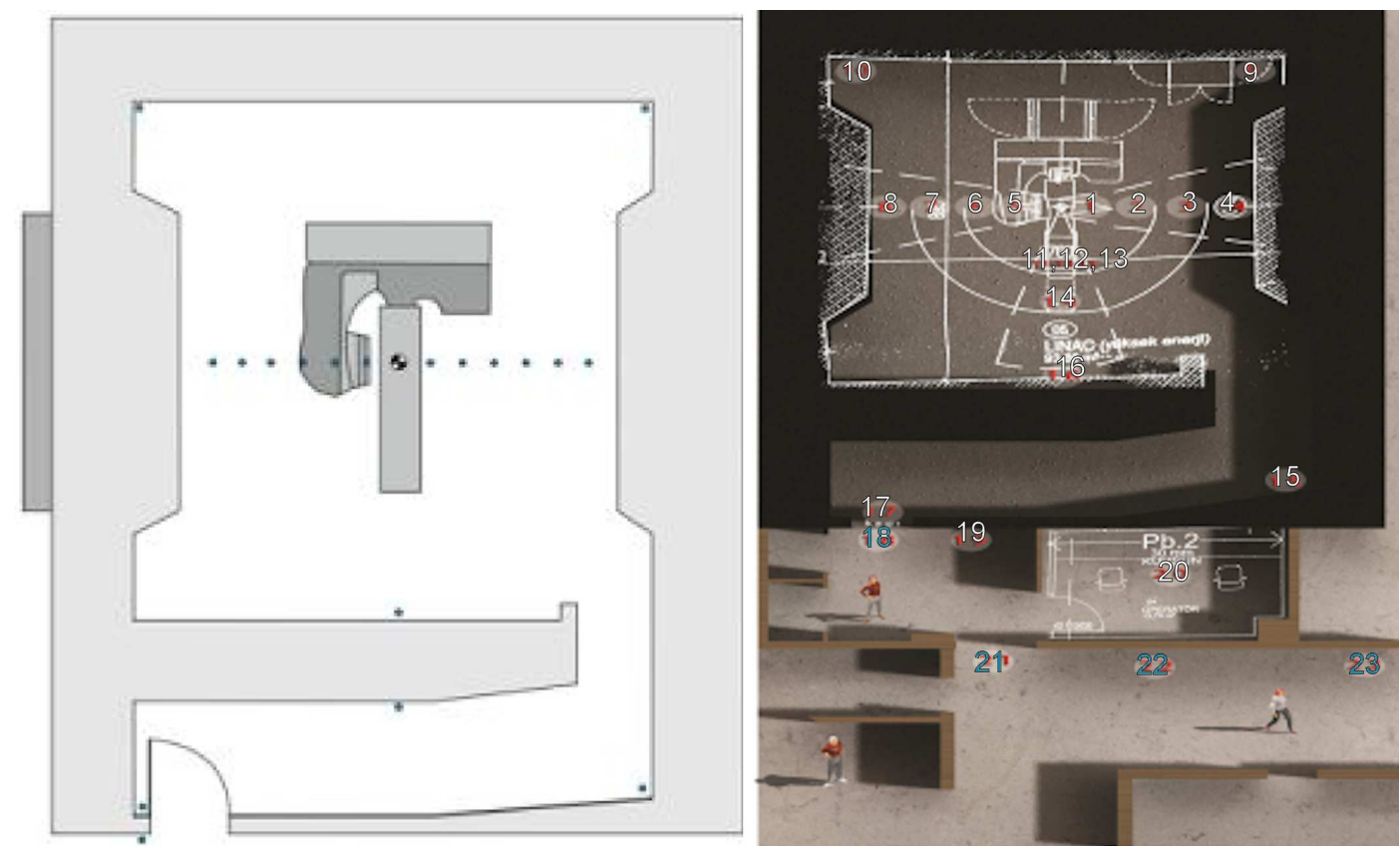

Fig. 1. Drawing and mock-up of measuring points in medical linac compartment.

*corresponding author; e-mail: umitkara@sdu.edu.tr 


\section{Material and method}

Radiation has existed since creation of universe and started to be used in a large of different fields nowadays. As human health is important one of the main used field of radiation is medical science for especially in the diagnostic and also treatment of diseases.

After chemotherapy treatment generally photon radiotherapy is commonly used method in cancer treatment. Photon is generated from the high-energy electron accelerators (LINAC) where an electron beam can be accelerated up to about $20 \mathrm{MeV}$. When electron beam impinged into thin metal photon is created via bremsstrahlung processes in medical hospital. The photoneutrons are produced in electron linear accelerators when the bremsstrahlung energy exceeds the threshold energy of photonuclear reactions of the materials $[1,3]$.

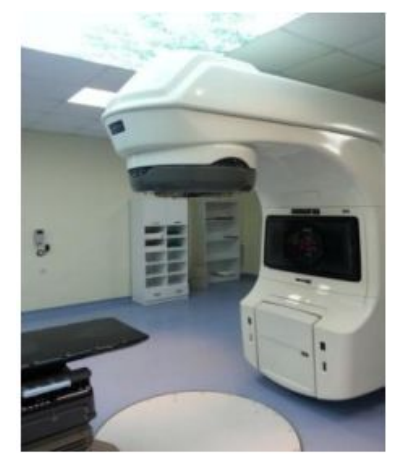

Fig. 2. Appliance used in measurements.

A significant number of neutrons are generated via $(\gamma, \mathrm{n})$ reaction. Those photoneutron contamination comes from the target, the filter, the collimator and any places struck by high energy X-rays [4-9]. Besides the known effects

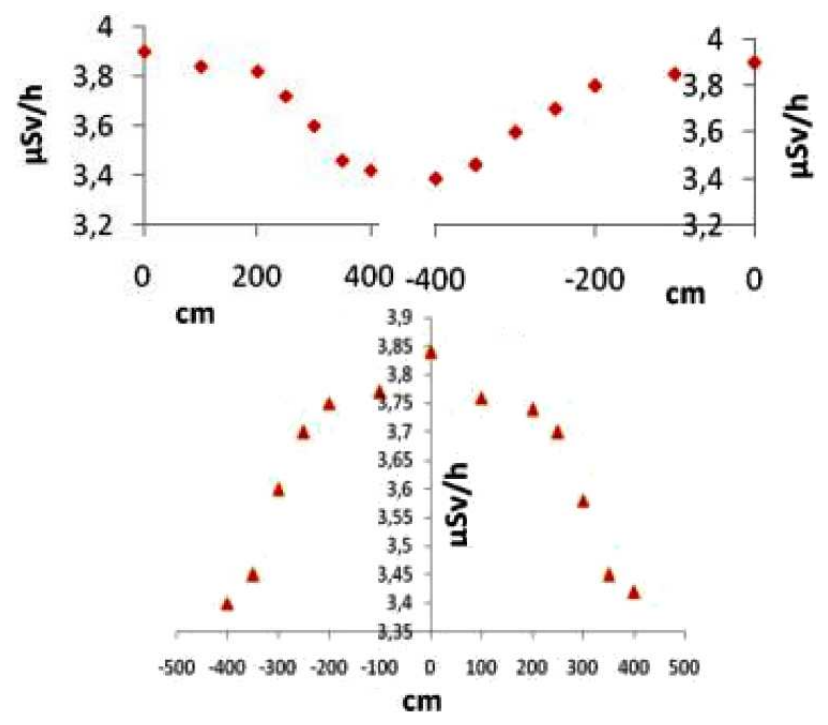

Fig. 3. Neutron dose $\left(0 \times 0 \mathrm{~cm}^{2}\right)$.

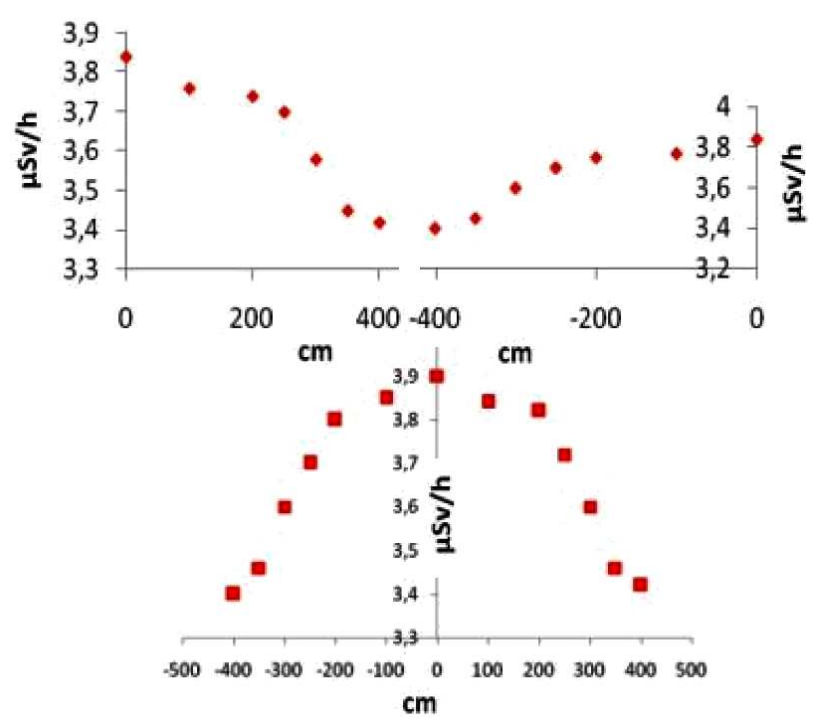

Fig. 4. Neutron dose $\left(20 \times 20 \mathrm{~cm}^{2}\right)$.

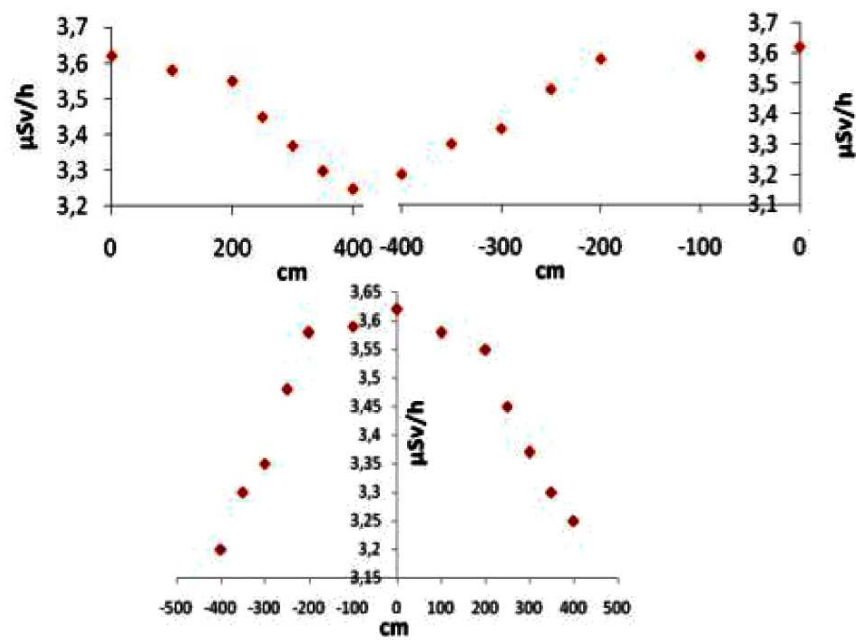

Fig. 5. Neutron dose $\left(40 \times 40 \mathrm{~cm}^{2}\right)$.

of radiation, the effects of surgical operations are still largely unknown. Thus it is important and also difficult to measure doses of physicians and their patients during radioatherapy treatment. In this work, radiation dose due to the neutron contamination of radiotherapy room has been calculated in using Monte Carlo methods.

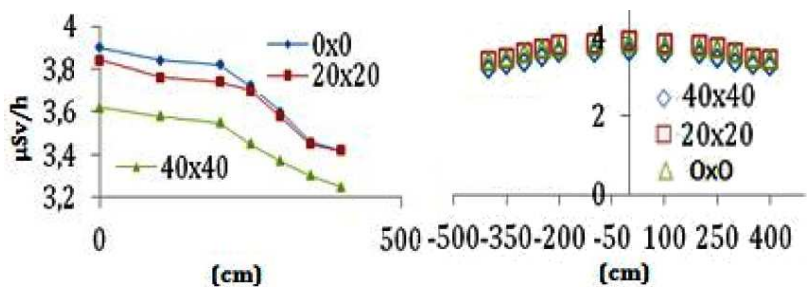

Fig. 6. The comparison of Monte Carlo method results. 


\section{Conclusion}

To get data for this paper we worked at Clinic Linear Accelerator (cLINAC) on Radiation Oncology Department in Suleyman Demirel Research Hospital. The threshold of the neutron production for these appliance is about $10 \mathrm{MeV}$. As the neutron is more dangerous than photon it is important to determine neutron dose during radiotherapy treatment. The neutron distribution depends on some parameters. In this study neutron dose rate has been simulated as a function of gantry angle. Results in points shown in Fig. 1 are gathered in Figs. 3-6.

In this study we simulated the dependency of cross sections for bremsstrahlung effect on photon spectra generated in the target and impinging on the surface of the phantom. However, the effect of these differences on dose computations are negligible.

\section{Acknowledgments}

This work has been supported partly by the Suleyman Demirel University Foundation Unit (3194-D212).

\section{References}

[1] The 2007 Recommendations of the International Commission on Radiological Protection, Ann. ICRP 37, 1 (2007).

[2] A.J. Lenox, Radiat. Phys. Chem. 61, 223 (2001).

[3] Neutron contamination from medical accelerators, NCRPÂ ReportÂ 79, 1984.

[4] I. Akkurt, J.O. Adler, J.R. Annand, F. Fasolo, K. Hansen, L. Isaksson, Phys. Med. Biol. 48, 3345 (2003).

[5] A. Mesbahi, Appl. Radiat. Isot. 67, 55 (2009).

[6] European Guidance on Estimating Population Doses from Medical Ray Procedures, Radiation Protection 154, European Commission, Luxembourg 2008.

[7] L. Paredes, R. Genis, M. Balcázar, L. Tavera, E. Camacho, Radiat. Meas. 31, 475 (1999).

[8] Fazal-ur-Rehman, H. Al-Ghamdi,Â M.I. Al-Jarallah, N. Maalej, Photoneutron Distributions around $18 \mathrm{MV}$ X-ray Radiotherapy Accelerators using Nuclear Track Detectors, King Fahd Unversity, Dhahran 2008.

[9] R.A. Geise, T.J. O'Dea, Appl. Radiat Isot. 50, 173 (1999). 